\title{
A novel quark-field creation operator construction for hadronic physics in lattice QCD
}

\author{
Michael Peardon* \\ School of Mathematics, Trinity College, Dublin 2, Ireland \\ John Bulava, Justin Foley, and Colin Morningstar \\ Department of Physics, Carnegie Mellon University, Pittsburgh, PA 15213, USA \\ Jozef Dudek, Robert G. Edwards, Bálint Joó, Huey-Wen Lin, and David G. Richards \\ Thomas Jefferson National Accelerator Facility, Newport News, VA 23606, USA \\ Keisuke Jimmy Juge \\ Department of Physics, University of the Pacific Stockton, CA 95211, USA \\ for the Hadron Spectrum Collaboration
}

(Dated: May 13, 2009)

\begin{abstract}
A new quark-field smearing algorithm is defined which enables efficient calculations of a broad range of hadron correlation functions. The technique applies a low-rank operator to define smooth fields that are to be used in hadron creation operators. The resulting space of smooth fields is small enough that all elements of the reduced quark propagator can be computed exactly at reasonable computational cost. Correlations between arbitrary sources, including multi-hadron operators can be computed a posteriori without requiring new lattice Dirac operator inversions. The method is tested on realistic lattice sizes with light dynamical quarks.

PACS numbers: 11.15.Ha,12.38.Gc,12.38.Lg
\end{abstract}

\section{INTRODUCTION}

One of the goals of lattice calculations is to predict the low-energy hadron spectrum of confined quarks and gluons, starting solely from the QCD lagrangian. This approach to spectroscopy necessitates methods for measuring the two-point correlation functions of field operators with the selected quantum numbers under investigation. A complete understanding of the QCD spectrum must also included excitations of the mesons and baryons as well as exotic states. Describing the resonances seen in scattering experiments as states in QCD has long presented a challenge to lattice calculations as direct access to the matrix elements related to decay widths is usually missing in Euclidean formulations of field theory. The way to circumvent this is well known in principle: decay properties can be inferred from a detailed study of the dependence of the spectrum in a finite box on the volume of the box [1, 2]. The best means of making solid determinations of energy levels is to employ a large basis of operators and then use the variational method to find good approximations to energy eigenstates. As soon as states above threshold are to be explored, the basis should include operators that resemble multi-hadron systems where the constituents have well-defined momenta. Access to all elements of the quark propagator [3] relevant to long-range correlation enables these measurements and also enables more detailed investigations into physical observables whose accurate measurement has often eluded lattice practitioners. One example is the isoscalar meson sector, where mass determinations require the evaluation of disconnected diagrams as part of the two-point correlation function. As well as this ambitious list of requirements, precision data will be crucial for these calculations [4].

In a Monte Carlo calculation on the lattice, the physically relevant signal in a correlation function falls exponentially and is rapidly dominated by statistical fluctuations. Operators that create low-lying energy eigenstates quickly are invaluable and improve the quality of data extracted exponentially. The most useful tool at the lattice practitioner's disposal in building good creation operators is smearing. The best means of understanding the low-energy degrees of freedom of confinement must thus come from a smearing method that can address all these features; it must be numerically accessible, do a good job of projecting onto the lowest energy states and it must facilitate easy evaluation of correlation functions involving a broad set of creation operators, including those exciting multi-hadron states.

*Electronic address: mjp@maths.tcd.ie 
A new generation of experiments devoted to hadron spectroscopy, including GlueX at Jefferson Lab, PANDA at GSI/FAIR and BES III intend to make measurements with unprecedented precision and in previously unexplored mass ranges and quantum-number sectors. The main aim of the Hadron Spectrum Collaboration is to extract from realistic lattice simulations predictions for masses, decay properties and relevant matrix elements in these domains.

Spectroscopy investigations are almost as old as non-perturbative Monte Carlo calculations on the lattice [5, 6]. As the first studies progressed, it was quickly realised that operators creating hadrons which are constructed directly from the fields in the lattice lagrangian have significant overlap with a large tower of states and that extracting the lightest elements of the spectrum was difficult. The solution was to build operators from "smeared" fields, where some linear operator was applied first to the quark degrees of freedom on the appropriate time-slice before the creation operator was formed. The aim of constructing a smeared field was to reduce the component of the fields close to the cut-off substantially, since these modes do not contribute significantly to long-range correlation functions. Initially, the techniques considered smeared fields that were not gauge covariant. Gauge-covariant schemes were introduced soon after [7, 8]. In particular, Ref. 8] introduced the Jacobi smearing algorithm, in which a lattice approximation to the three-dimensional gauge-covariant laplacian is constructed and applied iteratively to the field. This acts naturally as a long-wavelength filter on the modes constituting the quark field.

In this paper, a new method for smearing quark fields is described and tested in large-scale realistic simulations. In Sec. III the formulation of the new algorithm is presented, including detail on its application to measurements of hadron two-point correlation functions and three-point functions where an arbitrary current is inserted. Sec. III presents the results of initial tests of the effectiveness of the method, and Sec. IV] discusses practical issues that arise with the method and outlines some future research directions.

\section{OPERATOR CONSTRUCTION}

The energy of an eigenstate of the hamiltonian of a quantum field theory can be determined by computing the correlation function between creation and annihilation operators $\chi$ at Euclidean times $t$ and $t^{\prime}$;

$$
C\left(t^{\prime}, t\right)=\left\langle\chi\left(t^{\prime}\right) \chi^{\dagger}(t)\right\rangle
$$

Inserting a complete set of eigenstates of the hamiltonian, such that $\hat{H}|k\rangle=E_{k}|k\rangle$, this correlation function decomposes into a sum of contributions from all states in the spectrum with the same quantum numbers as the source operators,

$$
C\left(t^{\prime}, t\right)=\sum_{k}|\langle\chi \mid k\rangle|^{2} e^{-E_{k}\left(t^{\prime}-t\right)}
$$

In order to measure energies of low-lying states, it it crucial to construct operators that overlap predominantly with these light modes. This exposes the asymptotic behaviour of the correlator at earlier time-separations and enables more statistically accurate determinations of energies. There is a good deal of freedom in the construction of appropriate operators. Once the constraints of symmetries and temporal localisation are imposed, any function of the fields in the path integral can be used in principle.

Smearing is a well-established means of defining an initial step in the construction of creation operators. Rather than applying a creation operator to the fields directly, a smoothing function is applied first. The function should preserve as many symmetries as possible while effectively removing the presence of short-range modes, which make an insignificant contribution to the low-energy correlation function. In contemporary simulations, a popular form of this operation in theories where fermion fields couple to gauge bosons is the Jacobi smearing method [8].

Gauge-covariant quark smearings based on the lattice Laplacian start with the simplest representation of the second-order three-dimensional differential operator

$$
-\nabla_{x y}^{2}(t)=6 \delta_{x y}-\sum_{j=1}^{3}\left(\tilde{U}_{j}(x, t) \delta_{x+\hat{\jmath}, y}+\tilde{U}_{j}^{\dagger}(x-\hat{\jmath}, t) \delta_{x-\hat{\jmath}, y}\right),
$$

where the gauge fields, $\tilde{U}$ may be constructed from an appropriate covariant gauge-field-smearing algorithm [9]. After defining the Laplace operator, a simple smearing can be written

$$
J_{\sigma, n_{\sigma}}(t)=\left(1+\frac{\sigma \nabla^{2}(t)}{n_{\sigma}}\right)^{n_{\sigma}}
$$


where $\sigma$ and $n_{\sigma}$ are tunable parameters that can be used to optimise projection onto the states under investigation. For large $n_{\sigma}$, this approximates the exponential of $\sigma \nabla^{2}$, i.e.

$$
\lim _{n_{\sigma} \rightarrow \infty} J_{\sigma, n_{\sigma}}(t)=\exp \left(\sigma \nabla^{2}(t)\right) .
$$

The resulting exponential suppression of higher eigenmodes of the lattice Laplace operator means only a small number of the lowest modes contribute substantially to $J$.

This observation suggests the smearing operator can be approximated by forming an eigenvector representation, truncated to the lowest modes. Since the smearing operators used in lattice calculations are approximated well by low-rank constructions, a definition of smearing can be chosen to enforce this more absolutely. Let $V_{M}$ be the vector space of scalar fields charged under the fundamental representation of the gauge group on a particular time-slice. $V_{M}$ has rank $M=N_{c} \times N_{x} \times N_{y} \times N_{z}$ where $N_{c}$ is the number of colours, and $N_{x}, N_{y}$ and $N_{z}$ are the extents of the lattice in the three spatial directions. Now define smearing to be a well-chosen operator of rank $N \ll M$. This class of operators will be called "distillation" operators. There is a substantial benefit to doing this: if the rank of the operator is sufficiently small, all elements of the propagation matrix from this space can be constructed at an affordable computational cost (which is proportional to the rank of the space, $N$ ). Consequently, correlation functions involving arbitrarily intricate hadron creation operators can be measured with a fixed inversion overhead.

Define the distillation operator on time-slice $t$ as a product of an $M \times N$ matrix and its hermitian conjugate:

$$
\square(t)=V(t) V^{\dagger}(t) \Longrightarrow \square_{x y}(t)=\sum_{k=1}^{N} v_{x}^{(k)}(t) v_{y}^{(k) \dagger}(t)
$$

The $k^{\text {th }}$ column of $V(t)$ contains the $k^{\text {th }}$ eigenvector of $\nabla^{2}$ evaluated on the background of the spatial gauge fields of time-slice $t$, once the eigenvectors have been sorted by eigenvalue. This is the projection operator into $V_{N}$, the subspace spanned by these eigenmodes, so $\square^{2}=\square$. When the number of eigenvectors included is the same as the dimension of $V_{M}$, i.e. $N=M$, the distillation operator becomes the identity, and fields acted upon are unsmeared.

The Laplace operator inherits many symmetries of the vacuum. It transforms like a scalar under rotations, is covariant under gauge transformation and is parity and charge conjugation invariant. If the action of one of these symmetries on the Laplace operator maps $\nabla^{2}$ onto $\tilde{\nabla}^{2}$, then there is a unitary transformation, $R$ on $V_{M}$ such that

$$
R \tilde{\nabla}^{2} R^{\dagger}=\nabla^{2}
$$

This implies that if $v$ is an eigenvector of $\nabla^{2}$ then the eigenvectors of $\tilde{\nabla}^{2}$ are $R v$. Considering the definition of the distillation operator given in Eq. 6, the transformed operator must then obey

$$
R \widetilde{\square} R^{\dagger}=\square,
$$

and so correlation functions constructed using distilled fields have the same symmetry properties on the lattice as those constructed using Laplacian smearing methods.

\section{A. Meson two-point correlation functions}

Consider the momentum-projected creation and annihilation operators of an isovector meson, $\bar{u} \Gamma^{A} d$ and $\bar{d} \Gamma^{B} u$, where $\Gamma$ acts in spin and color as well as coordinate space. Applying the distillation operator $\square$ onto each quark field, the creation operator at three-momentum $\vec{p}$ is written as

$$
\chi_{M}^{\dagger}(\vec{p}, t)=\bar{u}_{x}(t) \square_{x y}(t) \cdot e^{-i p \cdot y} \Gamma_{y z}^{A}(t) \cdot \square_{z w}(t) d_{w}(t)
$$

where there is an implied summation over repeated spatial indices. In a shorthand notation the correlation function can be written as

$$
C_{M}^{(2)}\left(t^{\prime}, t\right)=\left\langle\bar{d}\left(t^{\prime}\right) \square\left(t^{\prime}\right) \Gamma^{B}\left(t^{\prime}\right) \square\left(t^{\prime}\right) u\left(t^{\prime}\right) \cdot \bar{u}(t) \square(t) \Gamma^{A}(t) \square(t) d(t)\right\rangle
$$

After evaluating the quark field path-integral and inserting the outer-product definition of the distillation operator $\square$ from Eq. 6. the correlator can be written

$$
C_{M}^{(2)}\left(t^{\prime}, t\right)=\operatorname{Tr}\left[\Phi^{B}\left(t^{\prime}\right) \tau\left(t^{\prime}, t\right) \Phi^{A}(t) \tau\left(t, t^{\prime}\right)\right]
$$


where

$$
\Phi_{\alpha \beta}^{A}(t)=V^{\dagger}(t)\left[\Gamma^{A}(t)\right]_{\alpha \beta} V(t) \equiv V^{\dagger}(t) \mathcal{D}^{A}(t) V(t) S_{\alpha \beta}^{A},
$$

and

$$
\tau_{\alpha \beta}\left(t^{\prime}, t\right)=V^{\dagger}\left(t^{\prime}\right) M_{\alpha \beta}^{-1}\left(t^{\prime}, t\right) V(t),
$$

with $M$ the lattice representation of the Dirac operator and where the quark spin indices, $\alpha, \beta$ of $\Phi$ and $\tau$ have been explicitly written. $\Phi$ has a well-defined momentum, while there is no explicit momentum projection in the definition of $\tau$. Often, $\Phi$ can be decomposed into terms that act only within coordinate and color space $\mathcal{D}^{A}$ and only within spin space $S^{A}$. Note that $\Phi$ and $\tau$ are square matrices of dimension $N \times N_{\sigma}$ where $N_{\sigma}$ is the number of components in a lattice Dirac spinor. Therefore it requires just $N \times N_{\sigma}$ operations of the inverse of the fermion matrix on a vector in order to compute all elements of $\tau$, the "perambulator". Notice also that the choice of source and sink operators is entirely independent of the computation of $\tau$; any source and sink operators can be correlated a posteriori once all elements of the $\tau$ matrix have been computed and stored. The method straightforwardly extends to the determination of correlation functions for mesons composed of different, non-degenerate quark flavors.

In the determination of an isoscalar meson correlation function, evaluation of disconnected terms is required. The disconnected diagram can be similarly represented once distilled fields are used in creation and annihilation operators. Such a term would comprise two separate traces over the distillation space:

$$
C_{M}^{(2, \text { disc })}\left(t^{\prime}, t\right)=\operatorname{Tr}\left[\Phi^{A}(t) \tau(t, t)\right] \operatorname{Tr}\left[\Phi^{B}\left(t^{\prime}\right) \tau^{\dagger}\left(t^{\prime}, t^{\prime}\right)\right] .
$$

An exact determination of this expression requires computing $\tau(t, t)$ for all time-slices $t$.

Since distilled single-particle operators can be projected onto definite momentum at both source and sink, multimeson correlators can also be constructed using creation and annihilation operators of the form

$$
\chi_{M M}\left(\vec{q}=\vec{p}_{1}-\vec{p}_{2} ; t\right)=\chi_{M}\left(\vec{p}_{1}, t\right) \chi_{M}\left(-\vec{p}_{2}, t\right),
$$

where $p_{1}$ and $p_{2}$ are the three-momenta of the single particle operators in Eq. 9. After integration over the quark fields, the resulting diagrams can again be computed by taking traces over products of construction operators in the distillation space with the perambulators. The precise structure of these functions depends on the quark flavor content of the source and sink operators, so details are not presented here. As before, these matrices are small compared to the dimension of the space of quark fields, and once the quark propagation is encoded in the perambulator, these multi-hadron correlation function can be computed.

\section{B. Baryon two-point correlation functions}

The factorization technique can also be applied to baryons. To illustrate the concepts involved, consider just the isospin- $1 / 2$ sector although the technique generalizes naturally to other baryon multiplets. An annihilation operator involving displacements as well as coefficients in spin space is written:

$$
\chi_{B}(t)=\epsilon^{a b c} S_{\alpha_{1} \alpha_{2} \alpha_{3}}\left(\mathcal{D}_{1} \square d\right)_{\alpha_{1}}^{a}\left(\mathcal{D}_{2} \square u\right)_{\alpha_{2}}^{b}\left(\mathcal{D}_{3} \square u\right)_{\alpha_{3}}^{c}(t),
$$

where the color indices of the quark fields acted upon by the displacement operators $\mathcal{D}_{i}$ are contracted with the antisymmetric tensor, and repeated spin indices are summed. After integration over quark fields, the correlation function

$$
C_{B}^{(2)}\left(t^{\prime}, t\right)=-\left\langle\chi_{B}\left(t^{\prime}\right) \bar{\chi}_{B}(t)\right\rangle
$$

can be factored into perambulator terms, Eq. 13 as well as creation and annihilation operators, where the latter can be written as

$$
\Phi_{\alpha_{1} \alpha_{2} \alpha_{3}}^{(i, j, k)}(t)=\epsilon^{a b c}\left(\mathcal{D}_{1} v^{(i)}\right)^{a}\left(\mathcal{D}_{2} v^{(j)}\right)^{b}\left(\mathcal{D}_{3} v^{(k)}\right)^{c}(t) S_{\alpha_{1} \alpha_{2} \alpha_{3}} .
$$

The spin terms factor from the antisymmetric contraction of the vectors $v$. 
There are two terms in the resulting correlation function that involve tensor contractions of the creation and annihilation operators with the perambulators

$$
\begin{aligned}
C_{B}^{(2)}\left[\tau_{d}, \tau_{u}, \tau_{u}\right]\left(t^{\prime}, t\right)= & \Phi^{(i, j, k)}\left(t^{\prime}\right) \tau_{d}^{(i, \bar{i})}\left(t^{\prime}, t\right) \tau_{u}^{(j, \bar{j})}\left(t^{\prime}, t\right) \tau_{u}^{(k, \bar{k})}\left(t^{\prime}, t\right) \Phi^{(\bar{i}, \bar{j}, \bar{k}) *}(t) \\
& -\Phi^{(i, j, k)}\left(t^{\prime}\right) \tau_{d}^{(i, \bar{i})}\left(t^{\prime}, t\right) \tau_{u}^{(j, \bar{k})}\left(t^{\prime}, t\right) \tau_{u}^{(k, \bar{j})}\left(t^{\prime}, t\right) \Phi^{(\bar{i}, \bar{j}, \bar{k}) *}(t),
\end{aligned}
$$

where there is an implicit tensor contraction over the internal spin indices, and where the quark labels $d$ and $u$ are used to denote the corresponding flavors of the quark perambulator terms in Eq. 13.

Similar to the meson case, the choice of source and sink operators is independent of the computation of the perambulators $\tau_{d}$ and $\tau_{u}$. The computation of these matrices can also be shared with computation of meson correlators. A baryon correlation function can be evaluated a posteriori using the contractions of the vectors with displacements in Eq. 18 which can also be shared among the source and sink operators. These contractions do not involve spin components, thus making the storage of $\Phi$ manageable.

\section{Meson three-point correlation functions}

A generic meson three-point function is written

$$
C^{(3)}\left(t_{f}, t, t_{i}\right)=\left\langle\left(\bar{d} \square \Gamma^{B} \square u\right)\left(t_{f}\right) \cdot(\bar{u} \Gamma u)(t) \cdot\left(\bar{u} \square \Gamma^{A} \square d\right)\left(t_{i}\right)\right\rangle,
$$

where there is no smearing in the operator on timeslice $t$. The completely connected Wick contraction can be expressed as

$$
C_{\mathrm{conn}}^{(3)}\left(t_{f}, t, t_{i}\right)=\operatorname{Tr}\left[\Phi^{B}\left(t_{f}\right) J\left(t_{f}, t, t_{i}\right) \Phi^{A}\left(t_{i}\right) \gamma_{5} \tau^{\dagger}\left(t_{f}, t_{i}\right) \gamma_{5}\right]
$$

where the "generalized perambulator" is defined by

$$
J_{\alpha \beta}\left(t_{f}, t, t_{i}\right)=V^{\dagger}\left(t_{f}\right)\left(M^{-1}\left(t_{f}, t\right) \Gamma(t) M^{-1}\left(t, t_{i}\right)\right)_{\alpha \beta} V\left(t_{i}\right)
$$

In general, a disconnected term may also appear; this can be factorised as the product of a two-point function (evaluated according to the algorithm of Sec. II A ) and a disconnected insertion. Since the disconnected trace does not involve distilled fields, the evaluation of this insertion is not discussed further at this point. To compute the connected three-point correlation function, there are two distinct sets of inversions needed. The first is from the distillation vectors at the source time-slice $t_{i}$ and the second from the sink time-slice $t_{f}$. The generalised perambulator of Eq. 22 is then constructed by contracting the two solutions from these two sets of inversions. The current insertion is encoded in the choice of operator $\Gamma$. Any momentum insertion at the current operator involves a Fourier transform on each time-slice $t$.

\section{Baryon three-point correlation functions}

Baryon three-point correlation functions can also be expressed in terms of the generalized perambulators of Eq. 22 . Consider a three-point correlation function where a baryon is created on time-slice $t_{i}$, then is acted on by a current operator on time-slice $t$ and is subsequently annihilated at $t_{f}$ :

$$
C_{B}^{(3)}\left(t_{f}, t, t_{i}\right)=-\left\langle B\left(t_{f}\right) \cdot(\bar{u} \Gamma u)(t) \cdot \bar{B}\left(t_{i}\right)\right\rangle
$$

After quark-field integration, this can be written as a sum of both a connected three-point contribution and the product of a disconnected insertion and a two-point correlator. As before, the discussion here is restricted to isospin$1 / 2$ light baryons although the technique is quite general. Note that the disconnected insertion involves unsmeared fields and must be evaluated through some other technique. The two-point contribution follows from Sec. IIB. For an up-quark insertion in the correlation function

$$
\begin{aligned}
& C_{\text {conn. }}^{(3)}\left(t_{f}, t, t_{i}\right)=-\epsilon^{a b c} \epsilon^{\bar{a} \bar{b} \bar{c}} S_{\alpha_{1} \alpha_{2} \alpha_{3}} \bar{S}_{\bar{\alpha}_{1} \bar{\alpha}_{2} \bar{\alpha}_{3}} \\
&\left\langle\left(\left(\mathcal{D}_{1} \square d\right)_{\alpha_{1}}^{a}\left(\mathcal{D}_{2} \square u\right)_{\alpha_{2}}^{b}\left(\mathcal{D}_{3} \square u\right)_{\alpha_{3}}^{c}\right)\left(t_{f}\right)\right. \\
&\left.\cdot(\bar{u} \Gamma u)(t) \cdot\left(\left(\bar{d} \square \overline{\mathcal{D}}_{1}\right)_{\bar{\alpha}_{1}}^{\bar{a}}\left(\bar{u} \square \overline{\mathcal{D}}_{2}\right)_{\bar{\alpha}_{2}}^{\bar{b}}\left(\bar{u} \square \overline{\mathcal{D}}_{3}\right)_{\bar{\alpha}_{3}}^{\bar{c}}\right)\left(t_{i}\right)\right\rangle,
\end{aligned}
$$


there are four Wick contractions after quark-field integration. The up-quark generalized perambulator from Eq. 22 is denoted as

$$
\widetilde{\tau}_{U}\left(t_{f}, t, t_{i}\right)=V^{\dagger}\left(t_{f}\right) M_{u}^{-1}\left(t_{f}, t\right) \Gamma(t) M_{u}^{-1}\left(t, t_{i}\right) V\left(t_{i}\right)
$$

and similarly for the down quark. Since the quarks in the insertion come in pairs, the connected three-point correlator can be written as the sum of two two-point correlation functions with each up-quark perambulator substituted with the corresponding generalized perambulator in turn;

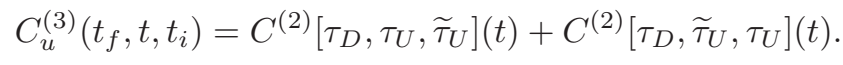

The down-quark contribution can be written as

$$
C_{d}^{(3)}\left(t_{f}, t, t_{i}\right)=C^{(2)}\left[\widetilde{\tau}_{D}, \tau_{U}, \tau_{U}\right](t) .
$$

This kind of construction is a generic feature of the three-point functions.

As in the previous two- and three-point correlator examples, the choice of source and sink operators is independent of the perambulator and generalized perambulator computations. These latter generalized perambulators can be shared among meson three-point correlator calculations. In addition, for degenerate quarks, only one generalized perambulator need be computed, independent of the flavor of the quark line where the current insertion is to occur. Thus, the overall computation of distinct three-point correlation functions has a manageable computational cost.

\section{TEST RESULTS}

To test the efficacy of the method, a first study of meson and baryon two-point correlators was performed. In addition, the method is demonstrated for a two-meson system. The background gauge fields were generated by the Hadron Spectrum Collaboration [10, 11]. They are $16^{3} \times 128$ and $20^{3} \times 128$ anisotropic lattices, with the ratio of spatial to temporal lattice spacings $a_{s} / a_{t}=3.5$ and $2+1$ flavors of dynamical quarks. The bare light-quark mass is $a_{t} m_{q}=-0.0840$, and when the $\Omega$ baryon mass is used to set the scale, the corresponding pion mass is $383 \mathrm{MeV}$. The valence-quark action is the same as the light-dynamical-quark action.

The numerical techniques for the iterative solution of the sparse linear system for the Sheikholeslami-Wohlert improved discretisation of the Dirac equation are described in Ref. [10]. In particular, the EigCG inverter [12] was used for the solution of these linear systems. This method uses eigenvector deflation to accelerate convergence. The first few inversion calls are needed to construct a sufficient basis, and subsequent inversions are accelerated by a factor of about three or more compared to the undeflated solver. For the lattices considered here, this asymptotic rate is found for $N \geq 4$.

\section{A. The profile of the distillation operator}

The distillation operator is written as a low-rank projection operator into a vector space of smooth fields. As such, there is little physical intuition to suggest it resembles more traditional smearing algorithms in generating a field that is roughly localized in some confining region while still remaining smooth. To examine the properties of the operator, its spatial distribution was computed. Defining

$$
\Psi(r)=\sum_{x, t} \sqrt{\operatorname{Tr}\left(\square_{x, x+r}(t) \square_{x+r, x}(t)\right)},
$$

means $\Psi$ is a gauge-invariant measure of the degree to which a field is smeared by the application of the distillation operator. This observable was measured for various values of $N$ and for a range of different off-set vectors $r$ on a small ensemble of the $20^{3}$ spatial lattices and the results are presented in Figure 1. The data clearly shows that for $N$ between 8 and 64 the distillation operator is very well described by a gaussian wavefunction. As $N$ increases, so the radius of this distribution reduces. This behaviour is to be anticipated since when $N=M$ the distillation operator is the identity and has no spatial extent at all. The measurements presented in Fig. 1 also clearly illustrate the distillation operator has good rotational symmetry, indicating it is only very weakly influenced by dynamics at the lattice cut-off. The artifacts seen at larger values of $r$ in the very broad $N=8$ distribution arise solely from the finite lattice volume used in these measurements. 


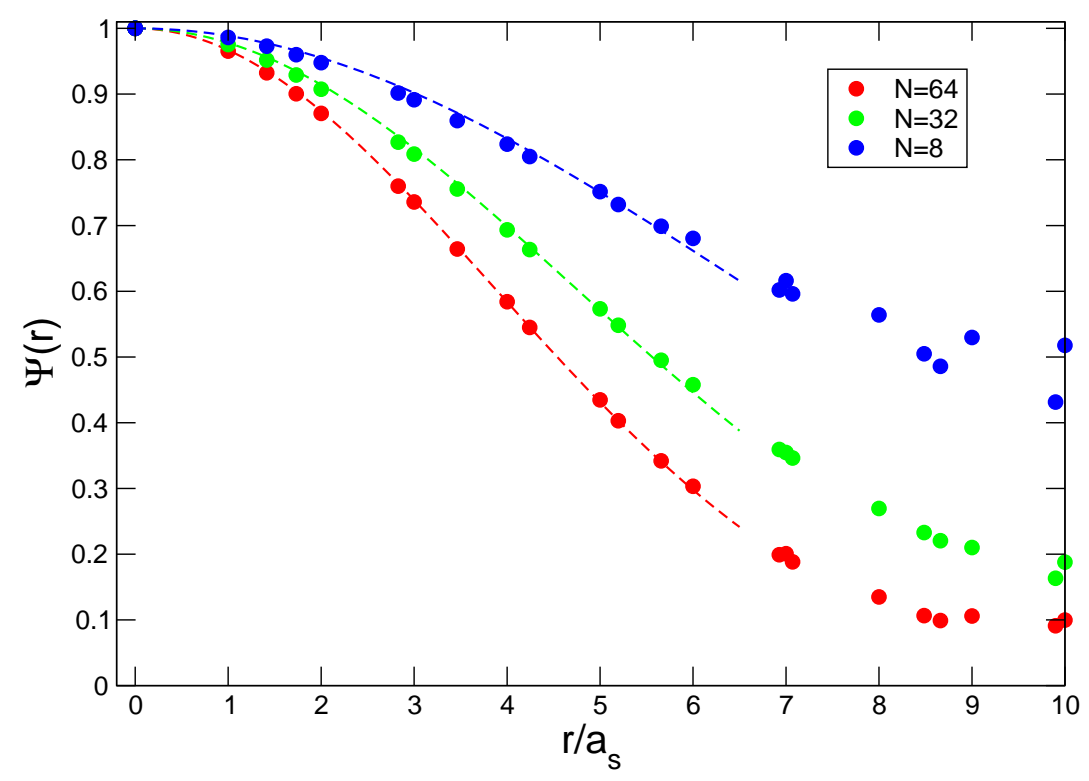

FIG. 1: The spatial distribution of the distillation operator constructed from the lowest eigenvalues of the Laplace operator on the lattice. Data is from a $20^{3}$ spatial volume and statistical fluctuations are much smaller than plot symbols. The dashed lines are fits to a gaussian distribution.

\begin{tabular}{c|c} 
Name & Construction \\
\hline$\gamma_{i}$ & $\bar{\psi} \gamma_{i} \psi$ \\
$a_{0} \times \nabla_{T_{1}}$ & $\bar{\psi} \nabla_{i} \psi$ \\
$\rho \times \mathbb{D}_{T_{1}}$ & $\left|\epsilon_{i j k}\right|\left|\epsilon_{k l m}\right| \bar{\psi} \gamma_{j} \nabla_{l} \nabla_{m} \psi$ \\
$\pi \times \mathbb{B}_{T_{1}}$ & $\epsilon_{i j k} \bar{\psi} \gamma_{5} \nabla_{j} \nabla_{k} \psi$
\end{tabular}

TABLE I: Derivative-based meson operators used in these tests. $\vec{\nabla}$ is the one-link discretised covariant derivative

\section{B. Meson correlation functions}

In this section, connected meson correlation functions formed using the derivative-based operators presented in Refs. 13, 14 are shown, with particular focus on the $T_{1}^{--}$channel. Fig 2 shows plots of the effective mass, defined by

$$
m_{\mathrm{eff}}(t)=-\frac{1}{\delta t} \ln \left(\frac{C(t+\delta t)}{C(t)}\right)
$$

with $\delta t=5 a_{t}$, for four diagonal correlators while varying the number of distillation vectors. Also shown are the corresponding effective masses computed using the traditional point-to-all propagator construction with both unsmeared and Jacobi-smeared sources. The four operators used are defined in Table \. In all cases, gauge links in the operators are stout-smeared [9]. 90 configurations of the $16^{3} \times 128$ lattice were used for this comparison.

A variational analysis [15, 16] of matrices of two-point correlation functions has proven to be a powerful method in extracting the spectrum and matrix elements of excited states [17]. Consequently, cost estimates in terms of the computing time required to form such a matrix of correlators and not just any single correlator should be considered. As a measure of the relative computational demands of distillation versus the traditional point-to-all approach, note that in the point-to-all approach, ten forward propagators, generated by applying $1, \nabla_{i=x, y, z},\left.\nabla_{i} \nabla_{j}\right|_{i \neq j}$ to a point source at the origin are required to produce the set of $T_{1}^{--}$correlation functions defined above. Each of these sources then requires twelve inversions of the Dirac matrix $\left(N_{c} \times N_{\sigma}\right)$. To compare with the distillation methods, note that approximately the same total inversion cost arises from using 32 distillation vectors since

$$
\left(N_{\mathrm{srcs}}=10\right) \times\left(N_{c}=3\right) \times\left(N_{\sigma}=4\right)=120 \approx(N=32) \times\left(N_{\sigma}=4\right)=128 .
$$

In the correlation function for the $\bar{\psi} \gamma_{i} \psi$ operator, it is clearly seen that increasing the number of vectors causes the effective mass to approach that of the unsmeared point-to-all correlation function, since $\sum_{k=1}^{M} v^{(k)} v^{(k) \dagger}=1$. The 



FIG. 2: Effective masses (five timeslice shift) for diagonal correlators in the $T_{1}^{--}$channel computed on $16^{3} \times 128$ lattices. The operator construction is shown in table \. Correlators are constructed using distilled quark fields with $N \in\{16,24,32,48,64\}$ vectors and using point-to-all methods with standard gauge-invariant quark smearing (SS, brown) and without (PP, black).

statistical variance is observed to decrease rapidly as the number of vectors is increased. For this range of $N$ it is always smaller than the point-to-all correlators. A similar but less rapid convergence of the signal is observed in the $a_{0} \times \nabla_{T_{1}}$ channel.

On the other hand, the $\pi \times \mathbb{B}_{T_{1}}$ signal appears to be essentially unchanged over this range of vector number but the noise reduces considerably with increasing vector number. The $\rho \times \mathbb{D}_{T_{1}}$ channel shows similar behaviour. Note that these operators sample non-trivial spatial dependence, and this may require a larger space of eigenvectors. The rapid scaling of noise with number of vectors can be quantified as follows: consider the correlator noise-to-signal ratio $r$ on a timeslice as a function of the number of vectors used, $N$ and model this behaviour with

$$
r=a+\frac{b}{N^{p}} .
$$

Fitting the parameters to best reproduce the Monte Carlo data from 450 configurations and averaging over many time-slices gives the following estimates for the scaling exponent

$$
p\left(\gamma_{i}\right)=0.8(1) ; \quad p\left(a_{0} \times \nabla_{T_{1}}\right)=1.1(2) ; \quad p\left(\pi \times \mathbb{B}_{T_{1}}\right)=1.3(3) ; \quad p\left(\rho \times \mathbb{D}_{T_{1}}\right)=1.8(2) .
$$

In all cases, the noise reduction is better than the behaviour expected from simple statistical scaling, $p=0.5$.

As a realistic test of the efficacy of distillation in the extraction of an excited-state spectrum, a matrix of correlation functions using twelve operators with $T_{1}^{--}$quantum numbers was constructed. In Fig. 3 the low-lying spectrum extracted as a function of number of vectors used is shown. Clearly, even with a relatively small number of vectors this method can be used to construct two-point correlation matrices from which the spectrum of low-lying states can be extracted with some precision. The masses, up through the fourth excited state, become more consistent with increasing number of vectors.

Now consider the issue of the scaling of this method with increasing lattice volume. Fig. 4 shows the effective masses of correlators computed on both $16^{3}$ and $20^{3}$ lattices, the spatial-volume ratio here being very close to 2 $\left(\frac{20^{3}}{16^{3}}=1.95\right)$. It is evident that the correlation function with $N$ vectors on the $16^{3}$ volume is essentially the same as the corresponding case with $2 N$ vectors on the $20^{3}$ lattice. Note that although the number of inversions for the same 


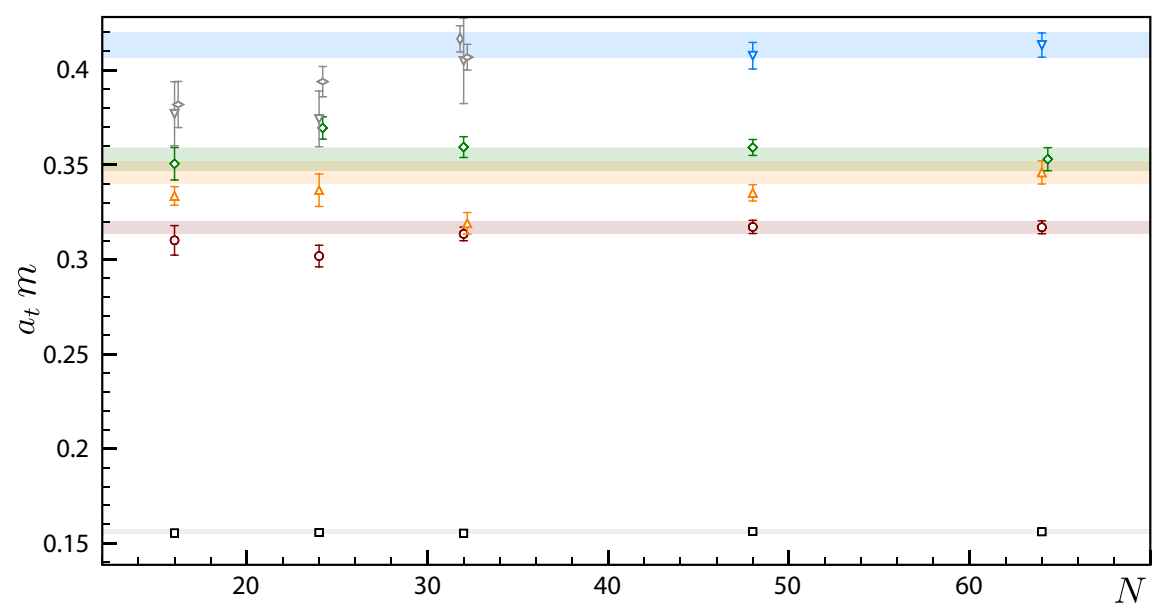

FIG. 3: $T_{1}^{--}$variational mass spectrum as a function of number of eigenvectors used. The error bars are purely statistical and do not take account of possible systematic errors due to fitting.

signal is doubled when the lattice volume increases from $16^{3}$ to $20^{3}$, there is a reduction in the noise of approximately a factor of two.

A strikingly simple explanation for this effect comes through consideration of the eigenvalues belonging to these

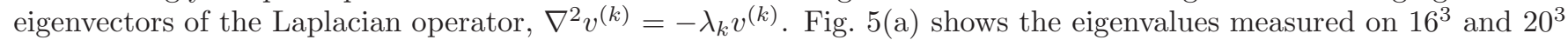
lattices. One could consider choosing the rank of the distillation operator by using all eigenvectors with eigenvalue below a certain cut-off. It is clear from the plot that on a larger volume this will require more eigenvectors. This is displayed more directly in Fig. 國(b) where the number of eigenvectors with eigenvalue less than a cut-off is plotted. Approximately twice as many eigenvectors in the $20^{3}$ case are required to have the same cut-off as in the $16^{3}$ case. It appears from this analysis that the meson correlator signal is effectively determined by the cut-off in eigenvalue used to construct the distillation space. This is equivalent to using a Heaviside approximation to smearing: $J_{\lambda}(t)=$ $\Theta\left(\lambda+\nabla^{2}(t)\right)=\sum_{k=1}^{M} \Theta\left(\lambda-\lambda_{k}\right) v^{(k)}(t) v^{(k) \dagger}(t)=\sum_{k=1}^{N(\lambda)} v^{(k)}(t) v^{(k) \dagger}(t)$.

\section{Baryon correlation functions}

Baryon correlation functions were evaluated using the displaced-quark operators described in Refs. 18, 19] and employed in spectrum studies Refs. 20, 21]. The distillation method is demonstrated on four nucleon $G_{1 g}$ operators, two of which are local to a single site with the remaining two having a singly displaced quark field. The corresponding $4 \times 4$ matrix of correlators was computed on 316 configurations of the $16^{3} \times 128$ lattice. Fig. [6] shows the effective-mass plots for the diagonal correlators. A clear trend is observed, similar to that seen for the mesons, of the correlator having larger excited-state contribution but smaller statistical fluctuations as the number of eigenvectors is increased. Modelling the correlator noise-to-signal ratio with Eq. 31 gives best-fit exponents $p \sim 1.1(2)$, showing again that increasing the number of vectors decreases the noise considerably faster than simple statistical scaling. The variational method [15, 16] was used to extract the masses of the lowest two states in the $G_{1 g}$ spectrum. Fig. 7 shows the dependence of the effective masses of these states on the number of eigenvectors used to form the distillation operator. Consistent masses are found, with an increase in statistical precision as the number of vectors is increased. Fig. 8 shows the diagonal effective masses computed on $16^{3}$ and $20^{3}$ lattices. The similarity between the signals found before and after doubling the number of vectors is not as obvious here as in the meson case. 

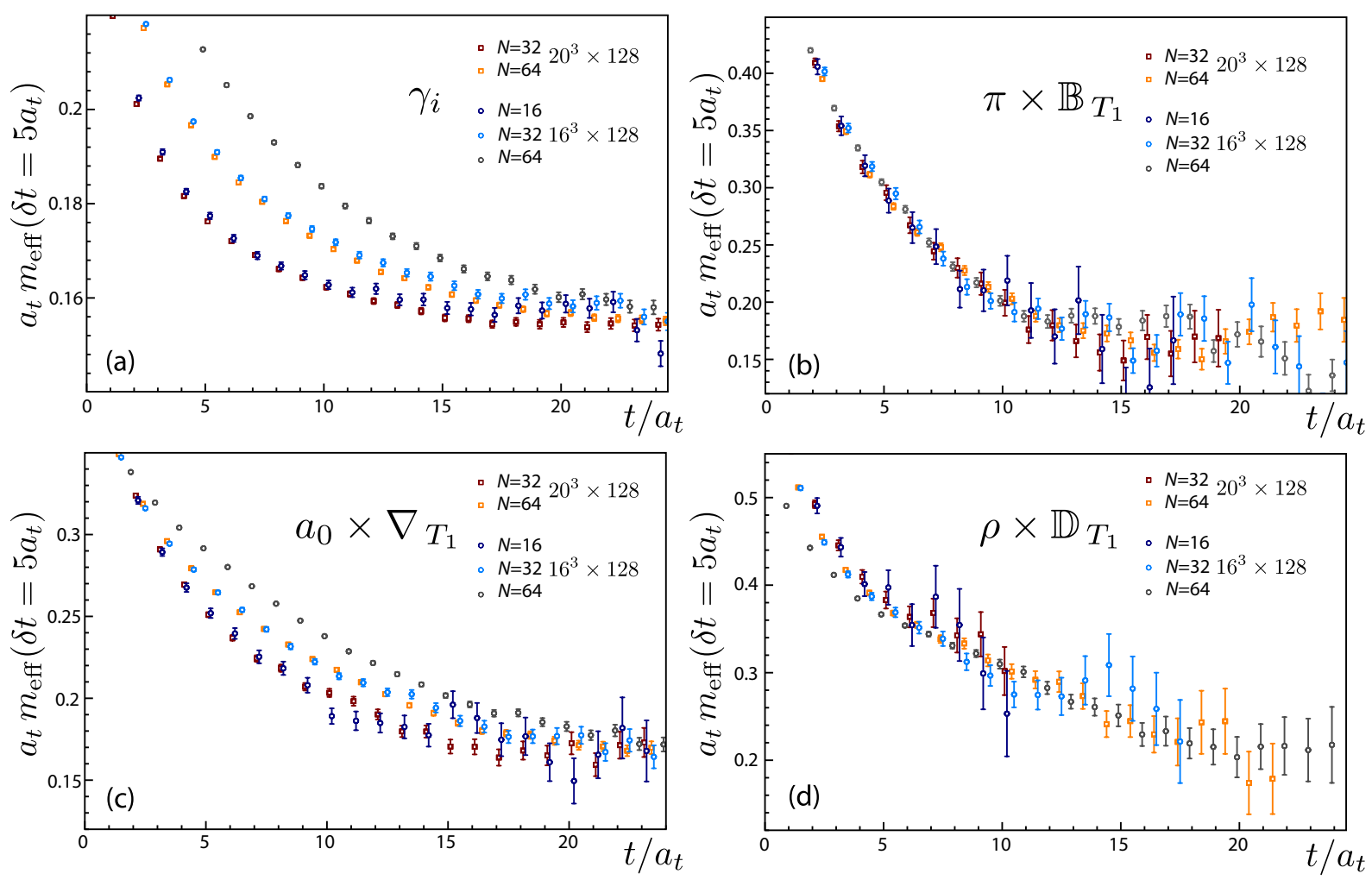

FIG. 4: Effective masses (five timeslice shift) for diagonal correlators in the $T_{1}^{--}$channel on $16^{3}$ and $20^{3}$ spatial volumes. Operator construction shown in table [
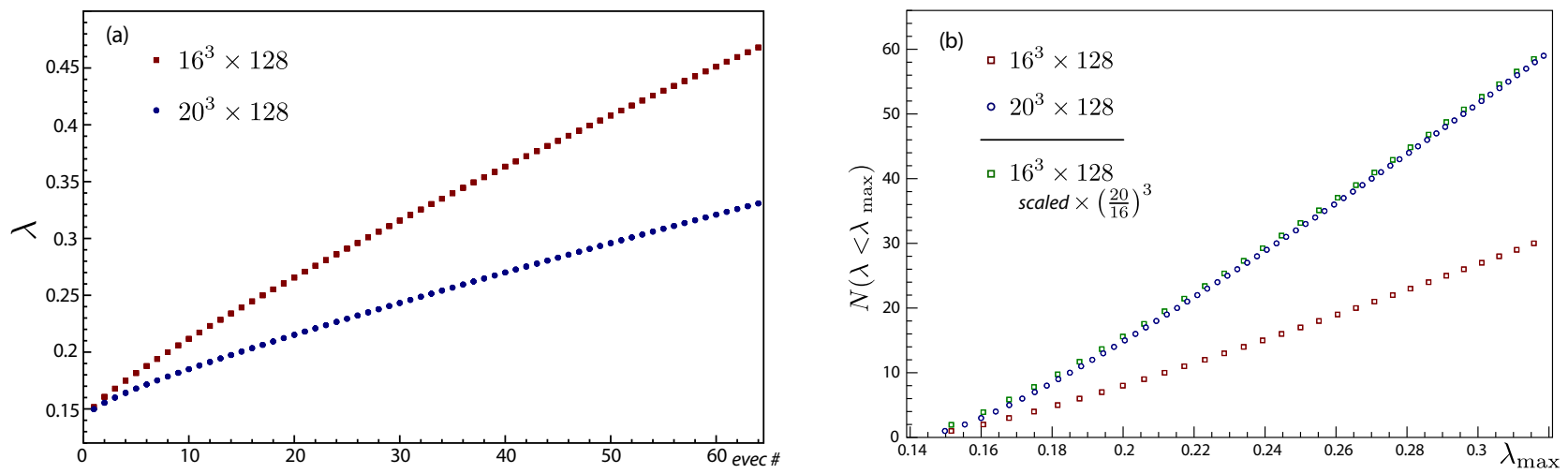

FIG. 5: (a) Eigenvalues of the laplacian on $16^{3}$ and $20^{3}$ lattices. (b) Number of eigenvectors with eigenvalue below $\lambda_{\text {max }}$ on $16^{3}$ and $20^{3}$. Also shown is the $16^{3}$ data scaled by a factor of $\left(\frac{20}{16}\right)^{3}$.

\section{Multi-meson correlation functions}

One of the main motivations for defining distillation methods is to enable efficient evaluation of correlation functions of creation operators which resemble multi-hadron states where the component hadrons have definite momentum. Reliable and efficient means of including these states in the operator basis are important for addressing scattering and resonance properties. The simplest case was tested, which comprises the isospin-two two-pion state formed using Eq. 15 with the single particle operators $M\left(\vec{p}_{\text {rel }}\right)$ constructed using the local operator $\bar{u} \gamma_{5} d$. For this example, consider the two-pion operator

$$
\chi_{M M}\left(\left|\vec{p}_{\text {rel }}\right|^{2}, t\right)=\sum_{k \in \mathcal{R}\left(\vec{p}_{\text {rel }}\right)} \chi_{M}(k, t) \chi_{M}(-k, t)
$$



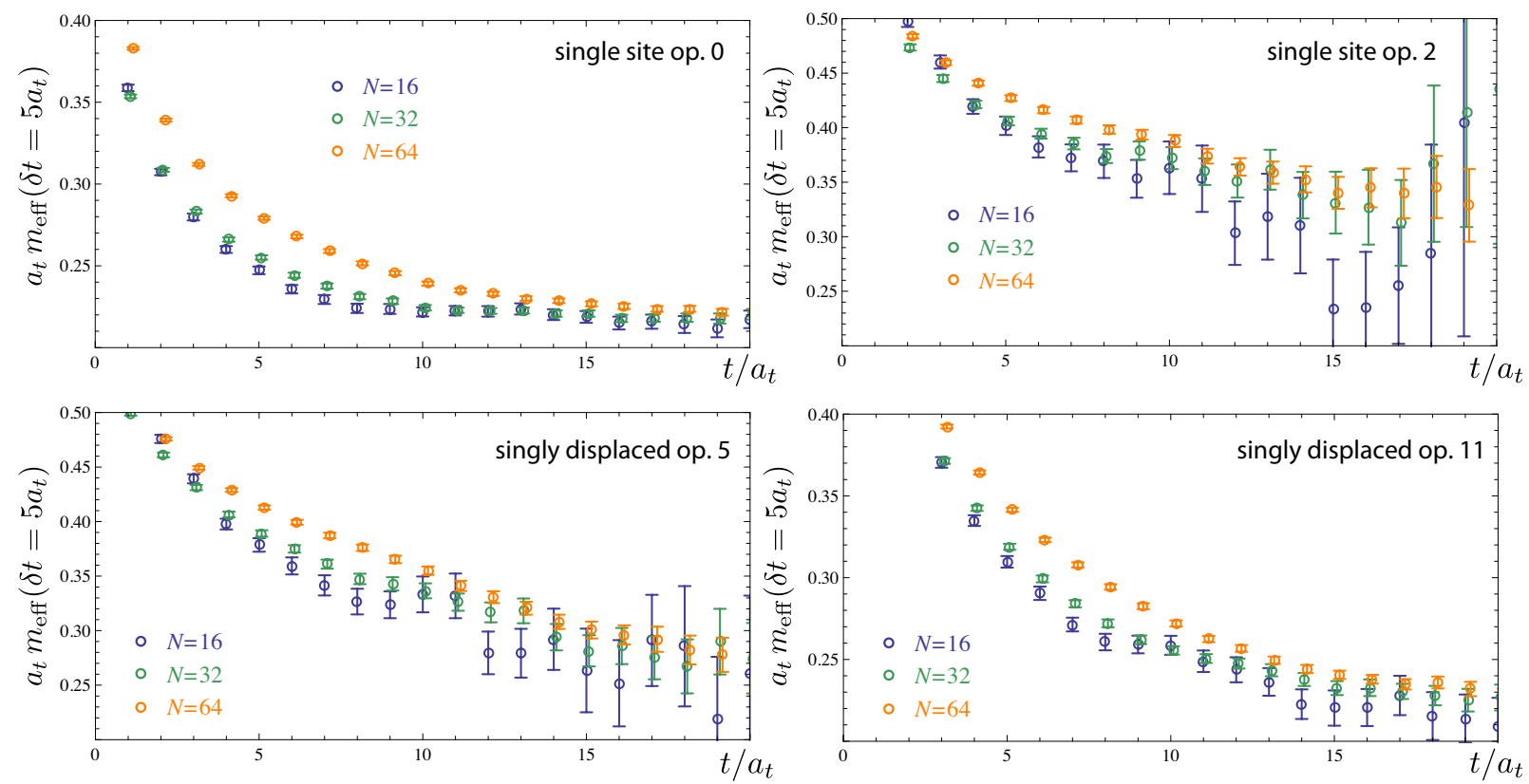

FIG. 6: Effective-mass plot for two single-site and two singly displaced operator correlators in the nucleon $G_{1 g}$ channel.

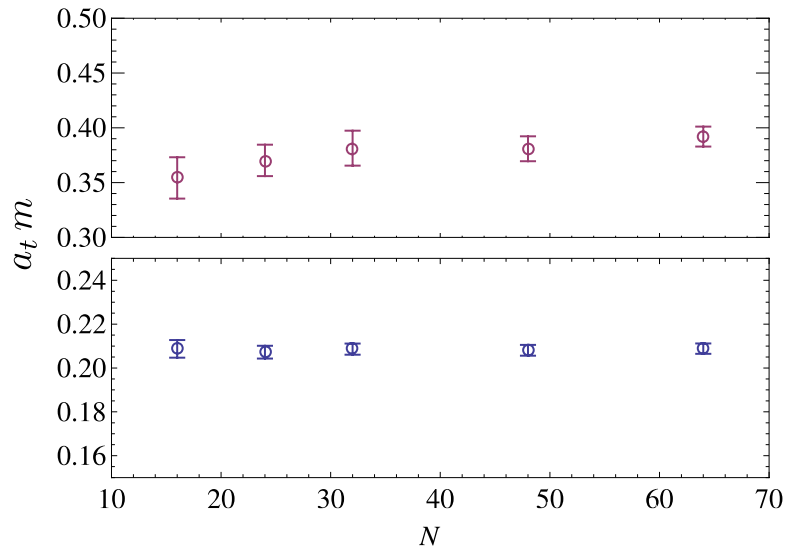

FIG. 7: The fitted energies for the first-excited (top) and ground state (bottom) as functions of $N$.

with total momentum zero, and the single-particle operators to have relative momentum $\vec{p}_{\text {rel }}$. The sum is over the set of momentum vectors $\mathcal{R}\left(\vec{p}_{\text {rel }}\right)$ related to $\vec{p}_{\text {rel }}$ by lattice rotations, to create a state that transforms trivially under rotations and so the operator forms an $A_{1}$ irreducible representation of the cubic group. In this test, all values of $\left|\vec{p}_{\text {rel }}\right|$ between 0 and $2 \times \frac{2 \pi}{L}$ were considered, corresponding to $\vec{p}_{\text {rel }}=\frac{2 \pi}{L} \vec{n}$ with $\vec{n} \in\{(0,0,0),(1,0,0),(1,1,0),(1,1,1),(2,0,0)\}$.

A $5 \times 5$ correlation matrix is formed between all of these two-pion operators which is diagonalized by solving the generalized eigenvalue problem. The effective mass of the lowest three principal correlation functions measured on 100 configuration is shown in Fig. 9. Clear indication of a signal for the second excited state is seen, even with this relatively low level of statistics. Note that when using the conventional point-to-all method, the source operator cannot be projected onto definite momentum so the correlation matrix described above cannot be constructed in practice. 

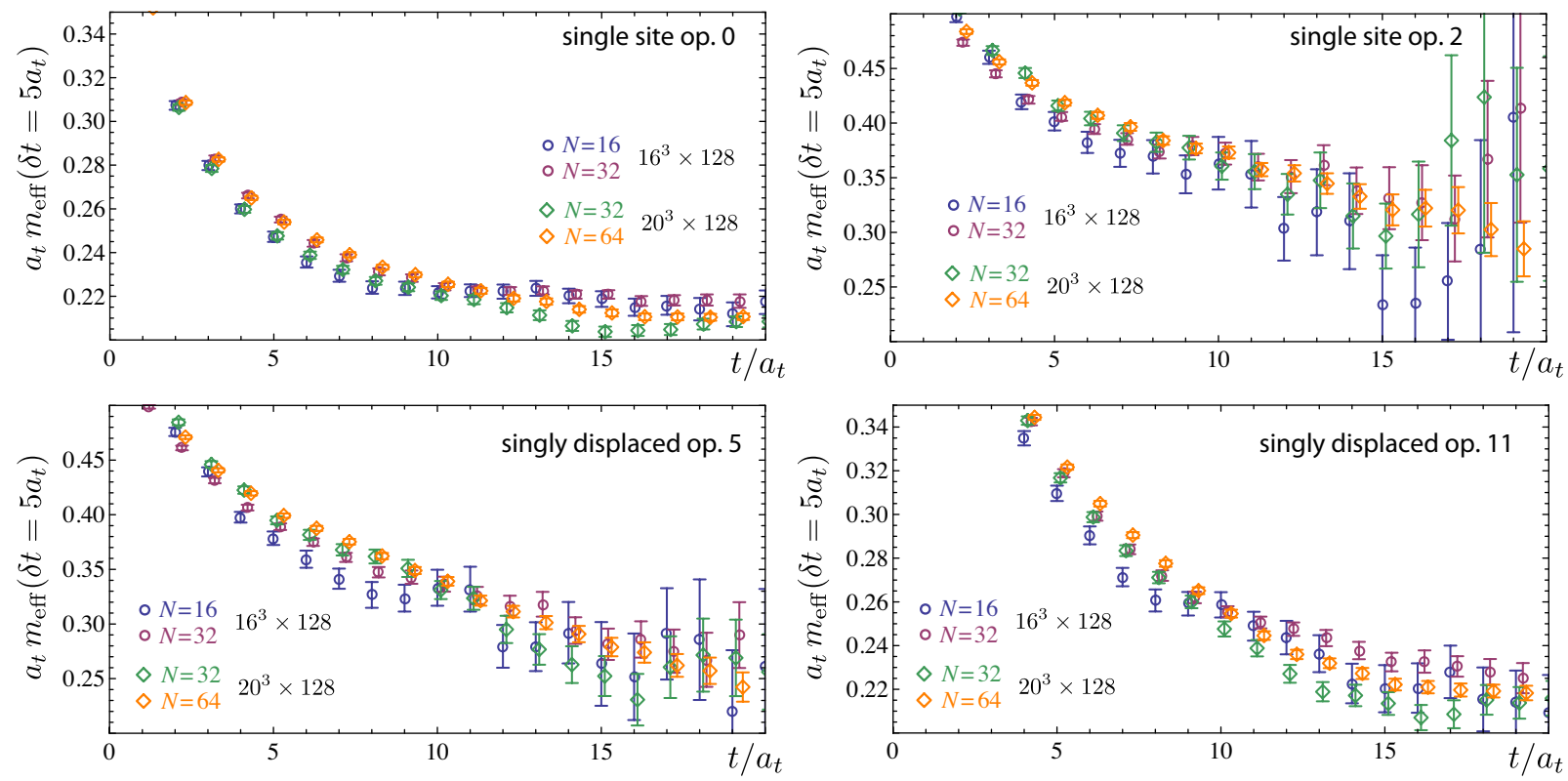

FIG. 8: Effective-mass plot for two single-site and two singly displaced operator correlators from two volumes: $16^{3} \times 128$ and $20^{3} \times 128$

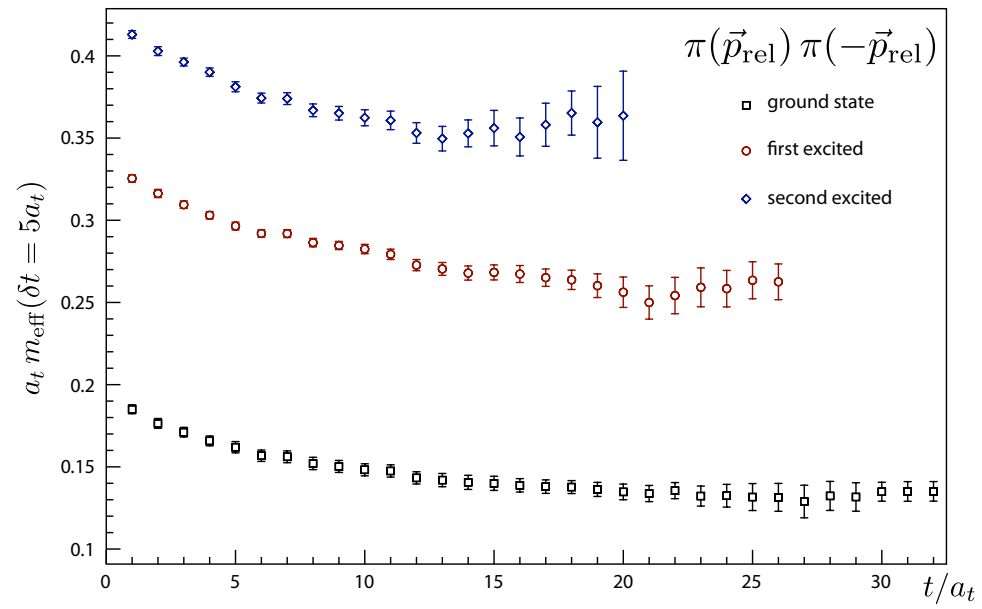

FIG. 9: Effective masses (five-timeslice shift) for the first three principal correlators in the isospin-two $\pi\left(\vec{p}_{\text {rel }}\right) \pi\left(-\vec{p}_{\text {rel }}\right)$ channel $\left(A_{1}^{++}\right)$computed on $16^{3} \times 128$ lattices. The $5 \times 5$ correlation matrix is constructed from $\left|\vec{p}_{\text {rel }}\right|^{2} \in[0,4]$. Correlators constructed through distillation with $N=64$ vectors on 100 configurations.

\section{DISCUSSION AND FUTURE DIRECTIONS}

The results of Sec. III demonstrate clearly that the light hadrons can be studied using operators that excite only a very small number of extended degrees of freedom on each time-slice. The restricted set of modes needed to create a hadron means all elements of quark propagation needed to construct the correlation function can be measured directly. This does not preclude using stochastic methods [3] to reduce computational costs.

In the straightforward recipe described in this paper, the distillation space was constructed from the lowest eigenvectors of the lattice three-dimensional Laplace operator on a time-slice. This definition is not unique and more experimentation with different choices of distillation space might prove useful. Also, the operator used in this work is diagonal in the quark spin index. Alternative choices of the distillation space might include non-trivial spin structure, using for example the vector space spanned by the lowest modes of the three-dimensional Dirac operator. 
Recall that in the simplest implementation tested here when $N=M$, the distillation operator becomes the identity and no smearing is performed. This is clearly an undesirable feature that is circumvented by the obvious variation on the method to include different weights for the eigenmodes. This more general distillation operator is then

$$
\mathcal{J}(t)=\sum_{k=1}^{N} v^{(k)}(t) f\left(\lambda_{k}(t)\right) v^{(k) \dagger}(t)
$$

Including weight functions on the eigenvectors can be used to suppress more rapidly fluctuating contributions when many eigenvectors are employed. Using exponential weights leads to increasingly accurate approximations to the conventional smearing algorithm of Eq. 4.

No increase in the number of eigenvectors would be expected as the continuum limit is taken at a fixed physical volume since, as is seen in the data of Sec. III A the smooth distilled modes are almost completely decoupled from the cut-off dynamics. This expectation is yet to be tested numerically. One substantial possible drawback with the method is the need to increase the rank of the distillation operator as the three-dimensional lattice volume increases. The corresponding linear increase in the cost of solving the Dirac equation leads to an algorithm for spectroscopy that scales like $\mathcal{O}\left(V^{2}\right)$. It appears from the results we have presented that with the required increase in rank with increasing volume one also obtains a decrease in statistical fluctuations. The possible solution to the issue of volume scaling may be to combine the method with a suitably designed stochastic estimation scheme. This is under investigation.

Note that the method does not give direct access to all elements of the quark propagator, only those relevant to low-energy spectroscopy. This would be a limitation in calculations involving isoscalar operator insertions, where

disconnected loops with all momentum components of the quark field are needed. A well-known example of such a computation would be the evaluation of the strangeness content of the nucleon.

\section{SUMMARY}

In this paper, a simple new quark-field smearing has been proposed and tested in numerical investigations using QCD gauge fields with $N_{f}=2+1$ dynamical flavors. There is substantial freedom in the construction of creation operators for hadrons. This has been exploited in this work to define an algorithm that applies a low-rank projection operator to the quark fields of the path integral on each time-slice in order to define smooth modes for subsequent operator construction. This makes affordable the evaluation of all information about quark propagation required to measure distilled hadronic correlation functions. Once the propagation matrix has been evaluated, correlations between arbitrary choices of creation and annihilation operators can be determined without requiring any further inversions. The theoretical framework needed to extend the scope of measurements using this technology to include matrix elements was described briefly.

Some initial tests of the method demonstrate that restricting quark fields to lie in a very low-dimensional space of smooth fields does not substantially degrade the quality of Monte Carlo evaluations of correlation functions and in many cases, statistical accuracy is enhanced. The most substantial drawback, still to be overcome, is the growth in cost of the algorithm as the lattice volume in physical units increases. Since the method is concerned with longdistance physics, no extra difficulties in approaching the continuum limit are anticipated, but this would need to be confirmed by practical testing.

The method is currently in its infancy and a number of research directions are outlined in this paper. The primary focus of the development efforts of this collaboration is to include stochastic evaluation of correlation functions in an optimal way and results will appear soon. The collaboration will use the techniques outlined in this paper in a range of spectroscopy determinations in the near future.

\section{Acknowledgements}

The Chroma software suite 22] was used to perform this work on clusters at Jefferson Laboratory using time awarded under the USQCD Initiative.

We thank Sinéad Ryan for many helpful comments on this manuscript. MP is supported by Science Foundation Ireland under research grant 07/RFP/PHYF168. MP is extremely grateful for the generous hospitality of the theory center at TJNAF during the early stages of this work. JB, JF and CM are supported by National Science Foundation grant numbers NSF-PHY-0653315 and NSF-PHY-0510020. KJJ is supported by grant number NSF-PHY-0704171.

Authored by Jefferson Science Associates, LLC under U.S. DOE Contract No. DE-AC05-06OR23177. The U.S. Government retains a non-exclusive, paid-up, irrevocable, world-wide license to publish or reproduce this manuscript 
for U.S. Government purposes.

[1] B. S. DeWitt, Phys. Rev. 103, 1565 (1956).

[2] M. Luscher, Nucl. Phys. B364, 237 (1991).

[3] J. Foley et al., Comput. Phys. Commun. 172, 145 (2005), hep-lat/0505023.

[4] C. Morningstar, arXiv:0810.4448 (2008).

[5] H. Hamber and G. Parisi, Phys. Rev. Lett. 47, 1792 (1981).

[6] D. H. Weingarten and D. N. Petcher, Phys. Lett. B99, 333 (1981).

[7] S. Gusken et al., Phys. Lett. B227, 266 (1989).

[8] C. R. Allton et al. (UKQCD), Phys. Rev. D47, 5128 (1993), hep-lat/9303009.

[9] C. Morningstar and M. J. Peardon, Phys. Rev. D69, 054501 (2004), hep-lat/0311018.

[10] R. G. Edwards, B. Joo, and H.-W. Lin, Phys. Rev. D78, 054501 (2008), arXiv:0803.3960.

[11] H.-W. Lin et al. (Hadron Spectrum), Phys. Rev. D79, 034502 (2009), arXiv:0810.3588.

[12] A. Stathopoulos and K. Orginos (2007), arXiv:0707.0131.

[13] X. Liao and T. Manke (2002), hep-lat/0210030.

[14] J. J. Dudek, R. G. Edwards, N. Mathur, and D. G. Richards, Phys. Rev. D77, 034501 (2008), arXiv:0707.4162.

[15] C. Michael, Nucl. Phys. B259, 58 (1985).

[16] M. Luscher and U. Wolff, Nucl. Phys. B339, 222 (1990).

[17] J. J. Dudek, R. G. Edwards, and D. G. Richards, Phys. Rev. D73, 074507 (2006), hep-ph/0601137.

[18] S. Basak et al., Phys. Rev. D72, 094506 (2005), hep-lat/0506029.

[19] S. Basak et al. (Lattice Hadron Physics (LHPC)), Phys. Rev. D72, 074501 (2005), hep-lat/0508018.

[20] S. Basak et al., Phys. Rev. D76, 074504 (2007), arXiv:0709.0008.

[21] J. M. Bulava et al., Phys. Rev. D79, 034505 (2009), arXiv:0901.0027.

[22] R. G. Edwards and B. Joo (SciDAC Collaboration), Nucl. Phys. B. Proc. Suppl. 140, 832 (2005), hep-lat/0409003. 